\title{
FINITENESS OF TOTAL CROSS-SECTIONS ${ }^{*}$ )
}

\author{
A. Martin \\ CERN--Geneva
}

\begin{abstract}
We derive an optimal condition for the finiteness of total cross-sections in potential scattering at any given energy, and by copying Froissart's trick, for elementary particles, explicit bounds on amplitudes and cross-sections in the spherically symmetric case. We also study the coupling constant dependence of the cross-sections for potentials of a given sign by using analyticity properties with respect to this coupling constant. This paper contains several new unpublished results.
\end{abstract}

Lectures given at the XX Universitätwochen für Kernphysik, Schladming, 1981.

*) to appear in Acta Physica Austriaca, Suppl.

Ref.TH. 3039-CERN

27 February 1981 


\section{INTRODUCTION}

In physics, we are familiar with situations in which the scattering amplitude and/or the total cross-section may be infinite. This is, for instance, the case with Rutherford scattering where the total cross-section and the forward amplitude are infinite, both in classical and quantum mechanics. However, for interactions which decrease much faster than the Coulomb interaction, a striking difference will appear between the classical and the quantum cases. In classical mechanics, if your interaction is not strictly vanishing beyond a certain distance, the total cross-section will be divergent, even for instance if $V(r)$, the potential, decreases exponentially. On the contrary, in quantum mechanics, we all know of simple cases where the total cross-section is finite, for instance scattering by a Yukawa potential $\exp (-\mu r) / r$. Naturally, one would like to know more than that and to see more clearly when the scattering amplitude is finite, including the forward direction, and when the total cross-section is finite. The interest of theoreticians in this problem is in waves. One of the first waves was in the sixties in the group of Professor Jauch in Geneva with some outsiders like myself. A renewal of interest has started recently with the paper of Amrein and Pearson ${ }^{1}$ ) which contains references to previous works. This paper stimulated my interest in the problem. Here at this school we have another of the main contributors, Volker Enss, who in papers with B. Simon ${ }^{2)}$ discusses both the finiteness and the coupling constant dependence of the total cross-sections by using the "geometrical methods" described here. Unfortunately, I shall be unable to present here a review talk and most of the time I shall restrict myself to my own approach. In particular. I shall not describe the seemingly very powerful method of Combes and Guez ${ }^{3)}$.

Maybe I should crudely describe the answers we should expect in three dimensions. To get a good partial wave scattering amplitude, we know that it is enough to have a potential decreasing somewhat faster than $1 / r$. To get a finite total cross-section, we need a potential decreasing somewhat faster than $1 / r^{2}$ and to get a finite forward scattering amplitude we need a decrease faster than $1 / r^{3}$.

Let me now describe the plan of my lectures. First I want to describe to you the strategy which was used by Froissart ${ }^{4)}$ to get a bound on elementary particle scattering amplitudes outside the framework of interactions by potentials. By adapting this method to the case of potentials, we derive bounds on amplitudes and total cross-sections. Then we describe a refined version of this approach in which both the bound and the finiteness are controlled by a unique quantity in the case of spherical symmetry. We also discuss the case of nonspherical symetry, first for total cross-sections averaged over the direction of the incident beam. We show that the same quantity controls the finiteness of 
the total cross-section and explain why we believe our condition to be optimal. We also prove strict finiteness without averaging over the incident direction, at the price of an extra condition. Generally speaking, we try to avoid the averaging process which seems necessary in the other methods of approach.

The last part of the lectures is devoted to a new, as yet unpublished, method of studying the coupling constant dependence of the total cross-section, which consists of using the analyticity and positivity properties of the forward scattering amplitude with respect to the coupling constant for a potential with one given sign. In some cases, this method gives the best possible accessible results. An amusing by-product is what we call a "Pomeranchuk theorem": we show that if we take a compact positive potential, which is known to give a total cross-section approaching the hard core cross-section for $g \rightarrow+\infty$, the corresponding hole obtained by taking $g$ negative produces a sequence of total crosssections for a sequence of $g^{\prime} s \rightarrow-\infty$ which also approach the hard core value.

2. THE METHOD OF FROISSART IN ELEMENTARY PARTICLE SCATTERING AND A POTENTIAL SCATTERING ANALOGUE

For a moment we shall leave the framework of potential scattering and look at the case of elementary particle scattering in which one extensively uses unitarity and analyticity to get a bound on the scattering amplitude and the total cross-section.

The scattering amplitude for two spin 0 particles can be written, with the relativistic normalization:

$$
F(s, \cos \theta)=\frac{\sqrt{s}}{2 k} \Sigma(2 l+1) f_{l}(s) P_{l}(\cos \theta)
$$

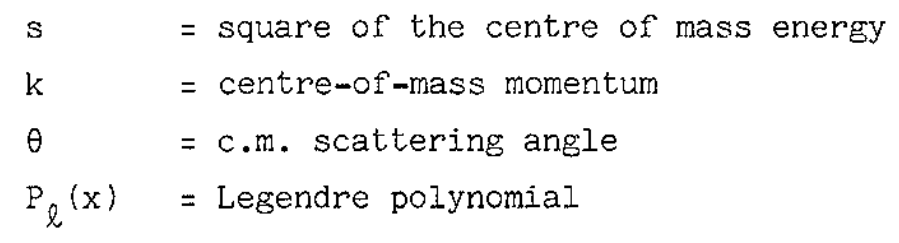

Unitarity is expressed by a condition on partial waves

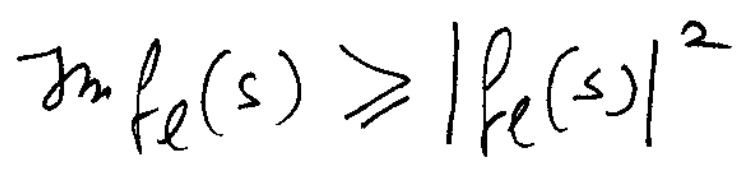

from which we get 
$-3-$

$0 \leqslant \operatorname{maf}(s) \leqslant 1$

(3)

at high energies, and

$$
\left|f_{l}(s)\right| \leqslant 1
$$

In $f_{\ell}(s)$ appears in the expression of the absorptive part

$$
A(s, \cos \theta)=\frac{\sqrt{s}}{2 k} \sum_{0}^{\infty}(2 l+1) \inf (s) P_{l}(\cos \theta),
$$

and via the optical theorem in the total cross-section

$$
\sigma_{\text {Total }}=\frac{2 \pi}{k \sqrt{s}} A(s, 1)=\frac{4 \pi}{k^{2}} \sum_{0}^{\infty}(2 l+1) y_{m} f_{l}(s) \cdot(6)
$$

Condition (3) is not sufficient to get a bound on (6) because we have an infinite sum. We need a control on large angular momentum partial wave amplitudes. Analyticity is the second ingredient we need. We demand that the absorptive part be continued beyond $\cos \theta=1$ with a convergent Legendre polynomial expansion. Specifically we need

$$
A\left(s, \cos \theta=1+\frac{4 m^{2}}{k^{2}}\right)<s^{2}
$$

where $m$ is a non-zero mass typical of the theory.

Inequality (7) has been obtained by Froissart by postulating the Mandelstam representation ${ }^{4}$. I have later shown that it is only a consequence of microcausality, positivity and the existence of a minimum mass in the theory ${ }^{5)}$. To get a bound on $I m f_{0}$ from (7), one uses the fact that each separate term of the series, being positive, is bound by $\mathrm{s}^{2}$ and a lower bound on Legendre polynomials: 
$-4-$

$$
P\left(1+\frac{4 m^{2}}{2 k^{2}}\right)>C_{\varepsilon} \exp \left((1-\varepsilon) \frac{2 m l}{k}\right), \forall \varepsilon>0 .^{(8)}
$$

So we get

$$
\begin{aligned}
& \operatorname{Im} f_{l}(s)<1, \\
& \operatorname{Im} f_{l}(s)<c_{1} s^{2} \exp \left(-\frac{c_{2} l}{k}\right),
\end{aligned}
$$

and we have to take the best of the two. It is easy to see that the critical value of $l$ for which we switch from (9) to (10) is

$$
L=C k \text { logs }
$$

Beyond $\mathrm{L}, \quad \mathrm{Im} f_{\ell}$ decreases exponentially and so does $f_{\ell}$, so we get

$$
\sigma_{\text {ToT }}<\frac{4 \pi}{k^{2}}\left(\sum_{0}^{L}(2 l+1)+\text { "Tail" }\right) \approx \text { Cost }(\log g)^{2}(12)
$$

and

$$
\begin{aligned}
& |F(s, \cos \theta)|<\operatorname{cosst} s(\log s)^{2} \\
& -1<\cos \theta<+1
\end{aligned}
$$

Now the parenthesis on elementary particle scattering is closed and we want to find an analogue in potential scattering to get bounds on amplitudes and total cross-sections. What I present here is an updated version of material containe in lectures I gave at the University of Washington in Spring 1964 6). We have, of course, to restrict ourselves to the case of spherically symmetric patenrials. We change the normalization of the amplitude: 
$-5-$

$$
\left.\begin{array}{c}
F\left(k^{2}, \cos \theta\right)=\frac{1}{k} \sum(2 l+1) f_{l}(s) P_{l}(\cos \theta) \\
I_{m} f_{e}(s)=\left|f_{l}(s)\right|^{2} \\
f_{l}(s)=e^{i \delta_{l}} \sin \delta_{e}, \delta_{e} \text { real }
\end{array}\right\}
$$

or

(15)

$$
\sigma_{\text {Total }}=\frac{4 \pi}{k} \quad A\left(k^{2}, \cos \theta=1\right)=\frac{4 \pi}{k^{2}} \sum(2 l+1) \operatorname{mm} f_{e}(s) .(16)
$$

The content of unitarity, expressed by (15), is no different from what we had in the relativistic case. What there remains to do is to find a condition which ensures that the partial wave amplitudes decrease for large $\ell$. Here we are interested in getting conditions as weak as possible on the long range decrease of the interaction. In fact, the most interesting application of these considerations is probably atomic and molecular physics. Therefore, we shall not impose the exponential decrease of the partial wave amplitude, which would, in fact, be equivalent to an exponential decrease of the potential.

The reduced, radial Schrödinger equation

$$
\left.\left(-\frac{d^{2}}{d r^{2}}+\frac{l(l+1)}{r^{2}}-k^{2}+v(r)\right) U_{l}(r)=0\right)
$$

(17)

can be transformed into the integral form

$$
u_{l}=j_{l}(k r)+\frac{1}{k} \int_{l}^{\infty} K_{l}\left(k r, \ell r^{\prime}\right) V \Gamma\left(r^{\prime}\right) v_{l}\left(r^{\prime}\right) d r^{\prime}
$$


$-6-$

where

$$
\begin{gathered}
j_{l}(x)=\sqrt{\frac{\pi x}{2}} J_{l+K_{2}}(x) \\
K_{l}\left(x, x^{\prime}\right)=\sqrt{\frac{\pi x}{2}} \sqrt{\frac{\pi x^{\prime}}{2}} J_{l+\xi_{\varepsilon}}(x<) H_{l+1_{\ell}}^{(1)}(x>)
\end{gathered}
$$

with $x<=x$ if $x<x^{\prime}, x<=x^{\prime}$ if $x>x^{\prime}$. I and $H$ are standard Bessel functions. The scattering amplitude is then given by

$$
e^{i \delta_{l}} \sin \delta_{l}=-\frac{1}{k} \int_{0}^{\infty} j_{l}(k r) V(r) u_{l}(r) d r .
$$

To study the integral equation (18), we use a separable bound on the kernel (20) which is an updated version of the bound obtained in Seattle 7 )

$$
\left|K_{l}\left(x, x^{\prime}\right)\right|<0.8 \frac{\sqrt{x x^{\prime}}}{(l+1 / 2)^{2 / 3}}
$$

This allows us to get an upper bound on the right-hand side of (18) in which the integral is factored out, and to obtain, after multiplication by $\sqrt{r} V(r)$ and integration

$$
\begin{aligned}
& \int\left|v_{e}(r)\right| V(r) \mid \sqrt{r} d r \\
& <\int\left|\dot{j}_{e}\left(l_{r}\right)\right| \sqrt{r}|V(r)| d r \\
& +\frac{0.8}{(\ell+1 / 2)^{2 / 3}} \int r|v(r)| d r \int\left|u_{e}(r)\right| V \mid \sqrt{r} d r,
\end{aligned}
$$

from which it is easy to get a bound on the scattering amplitude if the condition

$$
0.8(l+1 / 2)^{-2 / 3} \int r|V(r)| d r<1
$$


$-7-$

is satisfied. This bound is

$$
\left|e^{i \delta_{l} \sin \delta_{l}}\right|<\frac{\left|e^{i \delta_{l} \sin \sigma_{l}}\right|_{\text {BORN, }|V|}}{1-\frac{0.8}{(l+1 /)^{2 / 3}} \int_{0}^{\infty} r|v(r)| d r}
$$

with

$$
\left|e^{i \delta_{e}} \sin \delta_{l}\right|_{B O R N,|v|}=\frac{1}{k} \int\left|j_{e}(k r)\right|^{2}|V(r)| d r
$$

We see now that what replaces the critical $L$ of the relativistic case given by (11) is

$$
L=3\left[\int r|v(r)| d x\right]^{3 / 2}
$$

Then we get bounds on the total cross-section and the scattering amplitude, using $\left|f_{\ell}\right|$ and $\operatorname{Im} f_{\ell}<1$ for $\ell<L$ and (25) for $\ell \geq L$ :

$$
\begin{aligned}
& \left|F\left(k^{2}, \cos \theta\right)\right|<\frac{L^{2}}{k}+\frac{2}{k} \sum_{L}^{\infty}(2 l+1)\left|\sin \delta_{e}\right|_{\text {BORN, },|V|,}{ }^{(28)} \\
& -1 \leqslant \cos \theta \leqslant+1 \\
& \sigma_{\text {TOTAL }}<\frac{4 \pi}{k^{2}} L^{2}+\frac{8 \pi}{k^{2}} \sum_{L}^{\infty}(2 l+1)\left|\sin \delta_{e}\right|_{B O R N,(V) .}^{2(29)}
\end{aligned}
$$

In (28) and (29) the series (which are not at all negligible, as opposed to the relativistic case) can be majorized by replacing the sum from $L$ to $\infty$ by a sum from 0 to $\infty$. It is then possible to get closed expressions 
$-8-$

$$
\frac{1}{k} \sum_{0}^{\infty}(2 l+1)\left|\sin \delta_{l}\right|_{\text {BORN, }|V|}=\int \frac{d^{3} x}{4 \pi}|V(x)|,
$$

and

$$
\begin{aligned}
& \sum_{0}^{\infty}(2 l+1)\left|\sin \delta_{l}\right|^{2}= \\
& \int \frac{d \cos \theta}{2}\left[\sum(2 l+1) P_{l}(\cos \theta) \frac{1}{k} \int j_{l}^{2}(k r)|V| d r\right]^{2} \\
= & \int \frac{\left(\sin k\left|x-x^{\prime}\right|\right)^{2}}{\left(x-\left.x^{\prime}\right|^{2}\right.}|V(x)|\left|V\left(x^{\prime}\right)\right| \frac{d^{3} x}{4 \pi} \frac{d^{3} x^{\prime}}{4 \pi}=\frac{k^{2}}{4 \pi} \sigma_{\mathcal{B}} .
\end{aligned}
$$

In conclusion, we get the bounds

$$
\left|F\left(k^{2}, \cos \theta\right)\right|<\frac{9\left[\int r|v(r)| d r\right]^{3}}{k}+2 \int r^{2} / V(r) \mid d r(30)
$$

and

$$
\sigma_{\text {TOT }}<\frac{4 \pi}{k^{2}} \times 9\left[\int r / v(r) / d r\right]^{3}+4 I
$$

where

$$
I=\int \frac{|V(x)|\left|V\left(x^{\prime}\right)\right|}{\left|x-x^{\prime}\right|^{2}} \frac{d^{3} x}{4 \pi} \frac{d^{3} x^{\prime}}{4 \pi}
$$

In what follows, I will play an important role. If I is finite the potential belongs to the Rolinik class.

Let us remark that if $V=g v$ and if the various integrals exist, the bounds we have obtained imply 
$-9-$

$$
\left.\begin{array}{l}
\left|F\left(k^{2}, \cos \theta\right)\right|<\operatorname{cosect} g^{3} \\
\sigma_{\text {TOTAL }}<\cos s g^{3}
\end{array}\right\}
$$

(33)

It is easy to see that as far as the total cross-section is concerned, we need the convergence of $I$ and $r|V(r)| d r$, which is ensured if $V(r)$ decreases slightly faster than $\int r^{-2}$ at infinity. How much faster? We will postpone this question until the next section where we shall get a bound on $\sigma_{\text {total }}$ in terms of I alone.

3. AN "OPTIMAL" CONDITION FOR THE FINITENESS OF $\sigma_{T}$

Here we remain in the spherically symmetric case and consider only the problem of total cross-sections, not of amplitudes. We want to replace the two conditions of the previous sections by only one, which is the finiteness of $I$. The trick which was used in Ref. 8 ) is to find a bound on

$$
C_{e}(v)=\frac{1}{k^{2}} \int r^{2} d r r^{2} d r^{\prime}|V(r)| \mid K_{Q}\left(\left.\left(r, r^{\prime}\right)\right|^{2}\left|V\left(r^{\prime}\right)\right|\right.
$$

in terms of $I$ and $\ell$.

Suppose you can do this. Take Eq. (18), multiply by $\left|u_{\ell}\right||V|$ and integrate over $r$. Then use the Schwartz inequality in the right-hand side

$$
\begin{aligned}
& \int\left|v_{l}(r)\right|^{2}|V(r)| d r<\left[\int\left|v_{e}\right|^{2}|v| d r \int j_{e}^{2}|V| d r\right]^{1 / 2} \\
& +\int\left|v_{l}(r)\right|^{2}|V(r)| d r \times\left[C_{l}(V)\right]^{1 / 2}
\end{aligned}
$$

Then, if for large enough $\ell C_{\ell}(V)$ is less than one, you can keep the integral $\int u^{2}|v| d r$ under control and get bounds on the partial wave amplitude. In Ref. 8) we notice that

$$
K_{l}\left(|x|,\left|x^{\prime}\right|\right)=-\frac{i|x|\left|x^{\prime}\right|}{2} \int_{-1}^{+1} \frac{e^{i\left|x-x^{\prime}\right|}}{\left|x-x^{\prime}\right|} P_{l}(\cos \theta) \operatorname{des} \theta,
$$


$-10-$

where $\theta$ is the angle between $x$ and $x^{\prime}$. Hence, by Schwartz inequality

$$
\left|k_{e}\left(|x|,\left|x^{\prime}\right|\right)\right|^{2}<|x|^{2}\left|x^{\prime}\right|^{2} \int \frac{d \cos \theta}{2\left|x-x^{\prime}\right|^{2}} \cdot \frac{1}{2 \ell+1}
$$

which leads to

$$
C_{l}(V)<\frac{I}{2 l+1}
$$

However, while writing these notes the author realized that it is possible to appreciably improve (36). From the new estimates of Ref. 1 ) on $K_{\ell}\left(x, x^{\prime}\right)$ we have

$$
K_{l}(|x|,|x|)<0.8 \frac{\ln f\left(|x|,\left|x^{\prime}\right|\right)}{(l+1 / 2)^{2 / 3}}
$$

We now have

$$
\operatorname{lnf}\left(|x|,\left|x^{\prime}\right|\right)<\left[\frac{|x|\left|x^{\prime}\right| \mid}{2} \log \frac{|x|+\left|x^{\prime}\right|}{\| x|-| x^{\prime}||}\right]^{1 / 2}
$$

The bracket on the right-hand side of (39) is precisely proportional to the righthand side of (36) and we get

$$
\left|K_{l}\left(|x|, \mid x^{\prime}\right)\right|^{2}<\frac{0.64}{(l+1 / 2)^{4 / 3}}|x|^{2}\left|x^{\prime}\right|^{2} \int \frac{d \cos \theta}{2\left|x-x^{\prime}\right|^{2}}
$$

and hence

$$
C_{l}(v)<\frac{I \times 0.64}{(l+1 / 2)^{4 / 3}}
$$


- $11-$

So if $\ell>L_{0}$ given by

$$
L_{0}+L=(0.64 I)^{3 / 4}
$$

we get a bound on the partial wave amplitude

$$
\left|e^{i \delta_{l}} \delta_{\sin } \delta_{e}\right|<\frac{\left|e^{i \delta_{e}} \sin \delta_{e}\right| \text { BORN, }|V|}{1-0.8 \frac{\sqrt{I}}{(l+1 / 2)^{2 / 3}}}
$$

and from there we get a bound on the total cross-section

$$
\sigma_{T}<\frac{4 \pi}{k^{2}}\left(L^{2}+\frac{I}{\left(1-0.8 \frac{\sqrt{I}}{(L+1 / 2)^{2 / 3}}\right)^{2}}\right)
$$

for any $L$ integer $>L_{0}$. Taking for instance $L=I^{3 / 4}$ we get

$$
\sigma_{T}<\frac{4 \pi}{k^{2}}\left[I^{3 / 2}+25 I\right]
$$

Like the bound (3I) this corresponds to $\sigma_{t}<$ canst. $\times g^{3}$ if $V=g v$.

We claim that the finiteness of $I$ is an optimal condition. Naturally, we speak of the long distance part of the integrant. It is clear that if the potential contains hard obstacles in a compact region, this will not make the cross-section infinite. Suppose, however, that this is not the case. Suppose also that the potential is not oscillating. Then the Born approximation

$$
\frac{4 \pi}{k^{2}} \int \frac{\sin ^{2} k\left|x-x^{\prime}\right|}{\left|x-x^{\prime}\right|^{2}}\left|v\left(x^{\prime}\right)\right||V(x)| d^{3} x d^{3} x^{\prime}
$$


$-12-$

is not very different from I/2. If I diverges, the Born approximation diverges too and this may lead to an infinite total cross-section. We shall prove this in a specific case. Assume
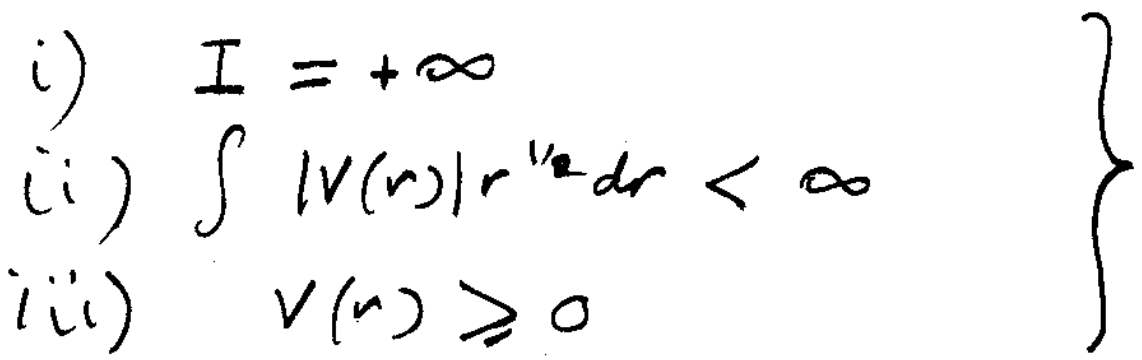

A potential like $\left(1+r^{1 \cdot 99}\right)^{-1}$ will satisfy these conditions. Under condition (i) one can still manage to control the large \& behaviour of partial wave amplitudes. Indeed, from the inequalities ${ }^{7)}$

$$
\begin{aligned}
& \left|K_{e}\left(x, x^{\prime}\right)\right|<.8(l+1 / 2)^{-2 / 3} \sqrt{x x^{\prime}} \\
& \left|K_{e}\left(x, x^{\prime}\right)\right|<1.2(l+1 / 2)^{1 / 3}
\end{aligned}
$$

one deduces

$$
\left|K_{e}\left(x, x^{\prime}\right)\right|<(l+1 / 2)^{-1 / 6}\left(x x^{\prime}\right)^{1 / 4}
$$

and, by the same machinery which leads to Eq. (23), one gets (for simplicity, we take here $k=1$ )

$$
\begin{gathered}
\int\left|v_{l}(r)\right||V(r)| r^{1 / 4} d r\left[1-(l+1 / 2)^{-1 / 6} \int|V(r)| r^{1 / 2} d r\right] \\
<\left[\int j^{2}(r)|V(r)| d r \int|V(r)| r^{1 / 2} d r\right]^{1 / 2}
\end{gathered}
$$

(47) 
$-13-$

from which it is not difficult to get, for

$e>\left(\operatorname{Siv}(r) \mid r^{1 / 2} d r\right)^{6}$

$$
\begin{aligned}
\left|\sin \delta_{l}\right| & >\left|\sin \delta_{l}\right|_{\text {BORN }} \\
& \times \frac{1-2(l+1 / 2)^{-1 / 6} \int r^{1 / 2} r(r) d r}{1-(l+1 / 2)^{-1 / 6} \int r^{1 / 2} V(r) d r}
\end{aligned}
$$

and hence taking $L=4096 \int V(r) r^{1 / 2} d r$

$\frac{k^{2}}{4 \pi} \sigma_{\text {TOT }}>-\frac{2}{3} \sum_{0}^{L}(2 l+1) \sin ^{2} \delta_{l B O R N}$

$+\frac{2}{3} \sum_{0}(2 l+1) \sin ^{2} \sqrt{l B O R N}$

Each sind $\delta_{\text {B Born }}$ is finite under the condition $i i$ ) in Eq. (45), but the last series, which, except for a factor $\sin ^{2}\left(\left|\vec{x}-\vec{x}^{\prime}\right|\right)$ in the integrant, coincides with $I$, is divergent. The only way to save the convergence would be to have an oscillating $V$ with carefully chosen oscillations. We believe that the technical assumption that $r^{1 / 2} \mathrm{~V}$ is integrable near the origin is inessential.

4. EXTENSION TO THE NON-SPHERICALLY SYMMETRIC CASE

First of all we must remember that if the potential has no symmetry, the total cross-section at a given energy will depend on the direction of the incident beam. It is clear for instance that if you take a flat disc, the cross-section will be maximum if the disc is perpendicular to the beam and very small (classically zero) if the disc is parallel to the beam. Under these circumstances, most authors introduce the notion of averaged total cross-section:

$$
\bar{\sigma}_{T}(k)=\int \frac{d \Omega_{k}}{4 \pi} \sigma_{T}(\vec{k})
$$


$-14-$

We shall also use this notion in the first part of this section, but later on we shall try to get conditions for the existence of $\sigma_{\mathrm{T}}$ for precise energy and direction of the incident beam.

Here we cannot eliminate the lower partial waves in which resonances can occur and therefore we shall modestly look for a situation in which the Born series converge.

We have

$$
\psi_{\vec{k}}(x)=\phi_{\vec{k}}(x)+\int \frac{d^{3} x^{\prime}}{4 \pi} \frac{e^{i k\left|x-x^{\prime}\right|}}{\left|x-x^{\prime}\right|} V\left(x^{\prime}\right) \psi_{\vec{k}}\left(x^{\prime}\right)
$$

Where $\phi_{\vec{k}}$ represents a plane wave or

$$
\psi_{\vec{k}}(x)=\phi_{\vec{k}}(x)+\int \frac{d^{3} x^{\prime}}{4 \pi} G_{k}\left(x, x^{\prime}\right) V\left(x^{\prime}\right) \phi_{k}\left(x^{\prime}\right)
$$

where $G_{k}\left(x, x^{\prime}\right)$ is the Green's function satisfying the integral equation

$$
G_{k}\left(x, x^{\prime}\right)=\frac{e^{i k\left|x-x^{\prime}\right|}}{\left|x-x^{\prime}\right|}+\int \frac{d^{3} x^{\prime \prime}}{4 \pi} \frac{e^{i k\left|x-x^{\prime \prime}\right|}}{\left|x-x^{\prime \prime}\right|} V\left(x^{\prime \prime}\right) G_{k}\left(x^{\prime \prime} x^{\prime}\right) .(53)
$$

The total cross-section is given by

$$
\sigma_{T}(\vec{k})=-\frac{1}{4 \eta} r_{m} \int^{-i \vec{k} \cdot x} V(x) C_{k}\left(x, x^{\prime}\right) V\left(x^{\prime}\right) e^{i k^{\prime}} d^{3} x d^{\prime} x^{\prime},
$$

and the average total cross-section by

$$
\sigma_{T}(k)=-\frac{1}{4 \pi k} \operatorname{Im} \int d^{3} x d^{3} x^{\prime} \frac{\sin _{k}\left|x-x^{\prime}\right|}{k\left|x-x^{\prime}\right|} V(x) G\left(x, x^{\prime}\right) V\left(x^{\prime}\right)
$$

Let us prove that if I, defined by Eq. (32), is less than unity, the Born series for $G$ converges. Take the square of the modulus of both sides of (53), multiply by $|V(\vec{x})|\left|V\left(\vec{x}^{\prime}\right)\right|$ and integrate. If we call 
$-15-$

$$
K=\int|v(x)|\left|G_{k}\left(x, x^{\prime}\right)\right|^{2}\left|v\left(x^{\prime}\right)\right| d^{3} x d^{3} x^{\prime}
$$

and repeatedly use Schwartz inequalities, we get

$$
K<I+2 I K^{1 / 2}+K I^{1 / 2}
$$

which implies, if $I<I$

$$
K^{1 / 2}<\frac{2 I^{1 / 2}}{1-I^{1 / 2}}
$$

On the other hand, application of the Schwarz inequality to (55) gives

$$
\begin{aligned}
\bar{\sigma}_{T}(k)< & \frac{4 \pi}{k} \sqrt{I k} \\
& <\frac{8 \pi}{k} \frac{I}{1-I^{1 / 2}} .
\end{aligned}
$$

The averaged total cross-section is therefore bounded if $I$ is less than unity.

We believe that in fact the condition $I<1$ is not really essential. If $I$ is finite, we know that $G_{k}\left(x, x^{\prime}\right)$ exists, except perhaps in a set of measure zero. Ins, during this series of lectures, pointed out to me that since what decides the finiteness of the potential is the outer part of the potential, one can always remove from the potential what is inside a sufficiently large sphere of radius $R$, so that $I<1$.

We would now like to discuss the finiteness of total cross-sections for a precise incident direction ${ }^{9)}$. If we expand the total cross-section in a series we see that the first term is

$$
-\frac{1}{k} \operatorname{Im} \int e^{-i \vec{k} x} V(x) \frac{e^{i k\left|x-x^{\prime}\right|}}{4 \pi\left|x-x^{\prime}\right|} V\left(x^{\prime}\right) e^{i \vec{k} \cdot x^{\prime}} d^{3} x d^{3} x^{\prime}
$$


$-16-$

$$
=\frac{1}{k} \int \cos \left(\vec{k} \cdot\left(x-x^{\prime}\right)\right) V(x) \frac{\left.\sin |k| x-x^{\prime}\right)}{4 n\left|x-x^{\prime}\right|} V\left(x^{\prime}\right) d^{3} x^{3} x^{\prime} \mid
$$

which is bounded (except for a factor $1 / k$ ) by

$$
J=\int|V(x)| \frac{1}{\left|x-x^{\prime}\right|}\left|V\left(x^{\prime}\right)\right| d^{3} x d^{3} x^{\prime}
$$

This indicates that we should supplement the condition I finite by $J$ finite if we want to guarantee that $\sigma_{T}(\vec{k})$ is finite without averaging. We shall now prove this statement in the case $I<I$ where the perturbation series for $G_{k}$ converges. Let us look at the $n^{\text {th }}$ term of the perturbation expansion of the cross-section. It is bounded by

$$
\sigma_{n}=\int\left|V\left(x_{1}\right) \frac{1}{4 n\left|x_{1}-x_{2}\right|}\right| v\left(x_{2}\right)|\ldots| v\left(x_{n}\right) \mid d^{3} x_{1} d^{3} x_{2} \cdots d^{3} x_{n}
$$

To majorize $\sigma_{n}$ in terms of $I$ and $J$, we multiply the integrant by

$$
\frac{\left|x_{1}-x_{2}\right|^{1 / 2}+\left|x_{2}-x_{3}\right|^{1 / 2}+\cdots+\left|x_{n-1}-x_{n}\right|^{1 / 2}}{\left|x_{1}-x_{n}\right|^{1 / 2}} \geqslant 1
$$

In this way, we get a sum of terms

$$
\begin{aligned}
\sigma_{n}< & \operatorname{Tr} H H \underbrace{k k \cdots k}_{n-2} \\
& +T_{2} H k H k \cdots k \\
& + \text { et z } \cdots
\end{aligned}
$$

with 


$$
\begin{aligned}
& H=|V(x)|^{1 / 2} \frac{1}{4 \pi\left|x-x^{\prime}\right|^{1 / 2}}\left|V\left(x^{3}\right)\right|^{1 / 2} \\
& K=|V(x)|^{1 / 2} \frac{1}{4 n\left|x-x^{\prime}\right|}\left|V\left(x^{1}\right)\right|^{1 / 2},
\end{aligned}
$$

and then

$$
\begin{aligned}
& x-2 T_{2}\left(H^{2}\right)\left(t K^{2}\right)^{\frac{n-2}{2}} \\
& =(n-2)(4 \pi)^{2} J^{\frac{n-2}{2}}
\end{aligned}
$$

so that $\sigma_{n}$ is finite if $J$ and $I$ are finite and, in addition, the series $\sum \sigma_{n}$ converges for $I<1$.

It is very difficult to know if the additional condition $J$ finite is too strong or not. If $\mathrm{V}$ is dominated by a spherically symmetric potential $\mathrm{W}$

$$
|V(x)|<W(|x|),
$$

I will be finite if $W(|\vec{x}|)$ decreases faster than $|\vec{x}|^{-2.5}$. This means that the condition $J$ finite is weaker than the requirement that $|V(x)|$ should be integrable. If $I$ is less than unity and $J$ finite, and if $\int V(x) d^{3} x$ diverges, we have a situation where the total cross-section is finite for any direction, but the forward amplitude is infinite. Indeed, it is trivial to see that the conditions $I<I$ and $J<\infty$ ensure not only the finiteness of the total crosssection, but also that of the scattering amplitude amputated from the Born term. If the Born term is infinite the forward amplitude is therefore infinite also.

5. THE COMPLEX $\mathrm{g}$ APPROACH TO TOTAL CROSS-SECTIONS

We now want to propose a new approach to study the growth of the potential with the coupling constant, based on analyticity properties of the forward scattering amplitude with respect to the coupling constant. We take the potential to be 


\section{$V=g v$}

and, for reasons which will become clear later, we take

\section{$v \leqslant 0$}

Then, if $\operatorname{Im} g>0, \operatorname{Im} V<0$. We shall, in addition, assume that

$$
I=\frac{1}{(4 \pi)^{2}} \int \frac{v(x) v\left(x^{\prime}\right)}{\left|x-x^{\prime}\right|^{2}} d^{3} x d^{2} x^{\prime}
$$

is finite and also in the first part we shall assume that the forward scattering amplitude exists:

$$
\int|v(x)| d^{3} x<\infty
$$

Then, the potential for Im $g>0$ is an absorptive potential, familiar to nuclear physicists who want to describe, for instance, neutron scattering by nuclei, with the special restriction that the real and imaginary parts are proportional. Everybody believes that such potentials are all right: the $S$ matrix exists, etc. I believe that it is not terribly difficult to prove rigorously that $F(g, \cos \theta=1)$, the forward amplitude, is analytic in the upper half plane in $g$. In the case of a spherically symmetric potential, the strategy I would use is to decompose into partial waves whose asymptotic form is

$$
e^{-i\left(k r-\frac{l n}{2}\right)}+\eta e e^{i\left(k r-l \frac{n}{2}\right)}
$$

and prove, using the Poincaré theorem, that $\eta_{\ell}$ is analytic in $g$. The absorptive character of $V$ guarantees that $\left|n_{\ell}\right| \leq 1$, and therefore that the $s$ matrix has no poles. It remains to resum the partial wave amplitudes. This may be tedious, but causes no real problem. This being said, the total cross-section is now made of two terms

$$
\sigma_{T}=\sigma_{\text {elastie }}+\sigma_{\text {Abs }} \text {. }
$$


$-19-$

$$
\sigma_{T}=\frac{4 \pi}{k} \operatorname{Im} F(g, \cos \theta=1)=-\frac{4 \pi}{k} y_{m} \int \phi_{\vec{k}}^{*} V \psi_{k} d^{3} x,(67)
$$

where $\phi_{k}$ is a plane wave and $\psi_{k}$ the exact wave function. $\sigma_{\text {elastic }}$ is clearly positive and

$$
\sigma_{A l s}=-\frac{4 \pi}{R} \int\left|\psi_{k}\right|^{2} I_{m} V d^{3} x
$$

is positive if In $V$ is $\leq 0$. This guarantees that the total cross-section is positive. The conclusion is that $F(g)$, forward scattering amplitude, is analytic in $\operatorname{Im} g \geq 0$ with $\operatorname{Im} F(g) \geq 0$. Such a function is called a Herglotz function. For a discussion of Herglotz functions I refer to the book of Shohat and Tamarkin, "The Problem of Moments" 10 ).

$$
\begin{aligned}
& \text { If } F \text { is a Herglotz function, it admits the representation } \\
& F(g)=A+B g+\frac{1}{\pi} \int_{-\infty}^{+\infty} \frac{\left(C+g g^{\prime}\right) I_{m} F\left(g^{\prime}\right)}{\left(C+g^{\prime 2}\right)\left(g^{\prime}-g\right)} d g^{\prime},
\end{aligned}
$$

with $A, B, C$ real

B>0, C>0, arbitrary,

and the properties

i) $\quad \frac{F(g)}{g} \rightarrow B \quad$ fo $g \rightarrow \infty, 0<A g g<\pi$

ii)

$$
\int_{-\infty}^{+\infty} \frac{j m F\left(g^{\prime}\right) \operatorname{dg}}{D^{2}+g^{\prime 2}}<\infty
$$

In our case, we can take $c=0$ because $\operatorname{Im} F(g)$ behaves like $g^{2}$ at $g \rightarrow 0$, and the representation reduces to 
$-20-$

$$
F(g)=A+B g+\frac{g}{\pi} \int_{-\infty}^{+\infty} \frac{7 m F\left(g^{\prime}\right) d g^{\prime}}{g^{\prime}\left(g^{\prime}-g\right)} .
$$

Property i) is precisely what we want. It shows that, in an averaged sense, $\sigma_{\mathrm{T}}(\mathrm{g})$ increases slower than $\mathrm{g}$, or more exactly $g(\log g)^{-1+\varepsilon}$.

Conclusion:

If

$$
\begin{aligned}
& \int|v| d^{3} x<\infty \\
& I<\infty \\
& \int \frac{\sigma_{T}\left(g^{\prime}\right) d g^{\prime}}{D^{2}+g^{\prime 2}}<\infty .
\end{aligned}
$$

(71)

The case where the forward amplitude is infinite.

If $F\left(g_{1} \cos \theta=1\right)$ is infinite, it remains that if $I$ is finite, what we can write as "F-F Born", averaged over incident directions, still exists. To make things precise, let us define $V_{\varepsilon}=V \exp (-\varepsilon r)$ with the intention of letting $\varepsilon$ go to zero. Then

$$
\lim _{\varepsilon \rightarrow 0}\left(F_{\varepsilon}-F_{\varepsilon \text { BorN }}\right)=\lim _{\varepsilon \rightarrow 0}\left(F_{\varepsilon}-g f_{\varepsilon \text { BORN }}\right)
$$

exists. We define

$$
\phi_{\varepsilon}=\frac{F_{\varepsilon}-g f_{\varepsilon \text { Bo eN }}}{g^{2}} \text {. }
$$

$\phi_{\varepsilon}$ exists at $g^{2}=0$. $\phi_{\varepsilon}$ behaves like $1 / g$ for $g$ going to $\infty$ in complex directions and therefore satisfies the integral representation

$$
\phi_{\varepsilon}=\frac{1}{\pi} \int_{-\infty}^{+\infty} \frac{J_{m} F_{\varepsilon}\left(g^{\prime}\right) d g^{\prime}}{g^{\prime 2}\left(g^{\prime}-g\right)}
$$


$-21-$

On the other hand, $\phi_{\varepsilon} \rightarrow \phi$ for $\varepsilon \rightarrow 0$, at least inside the (finite) radius of convergence of the power series of the amplitude in $g$ around $g=0$; (the radius is $>0$ because $I<\infty)$. Take the imaginary part of (73) for some comflex value of $g$ inside the radius of convergence. We have

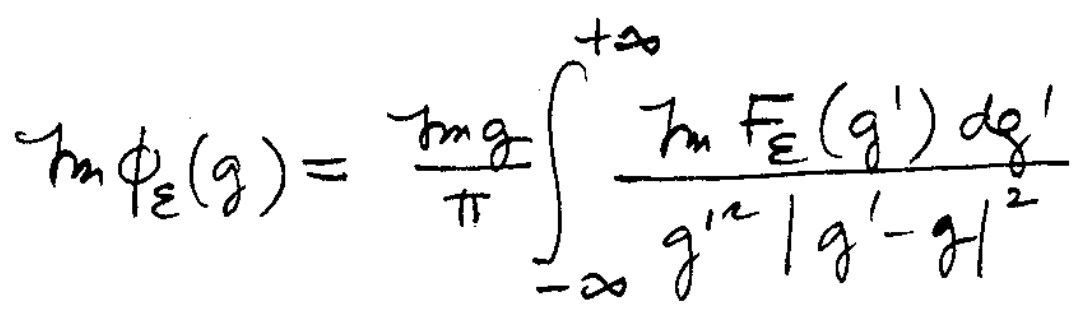

$\operatorname{Im} \phi_{\varepsilon}(g) \rightarrow \operatorname{Im} \phi(g)$ for $\varepsilon \rightarrow 0$. On the other hand, notice that the integrant in (74) is positive. The integrant has a limit as $\varepsilon \rightarrow 0$. We are in a case of dominated convergence and

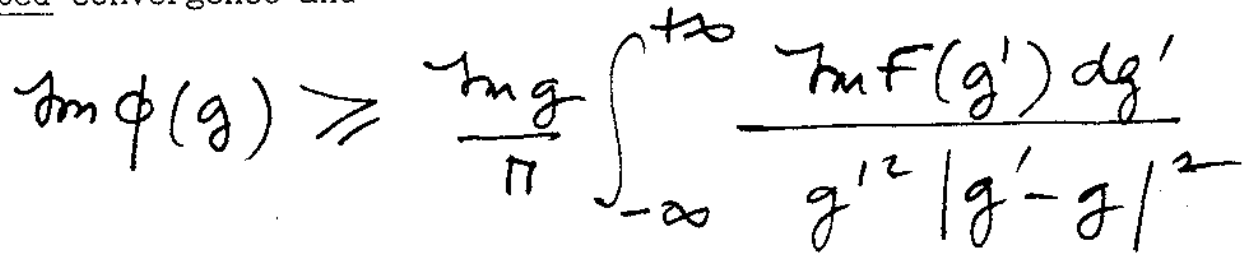

The conclusion is

$$
\int \frac{\sigma_{\text {Toll }}\left(g^{\prime}\right) d g^{\prime}}{g^{\prime 2}\left(g^{\prime 2}+c^{2}\right)}<\infty
$$

This corresponds to an average increase slower than $g^{3}(\log g)^{-1}$. This result is not as good as that of Ens and Simon for potentials decreasing like $r^{-2-\varepsilon}$, which is that $\sigma_{\text {total }}$ cannot increase faster than $8^{2}$. However, the condition $I<\infty$ contains potentials behaving like $r^{-2}(\log r)^{-1 / 2-\varepsilon}$. It is not yet exclued that for such potentials one could have such an increase for particular energies.

Finally, we want to illustrate the use of this complex \& technique by an "analogue" of the "Pomeranchuk theorem". Let me remind you that in elementary particle scattering, the particle-particle amplitude and particle-antiparticle amplitudes are connected by crossing symmetry and correspond to boundary values of the same analytic function for energies approaching $\pm \infty$. Then, the "Pomeranchuk theorem" says that 
$-22-$

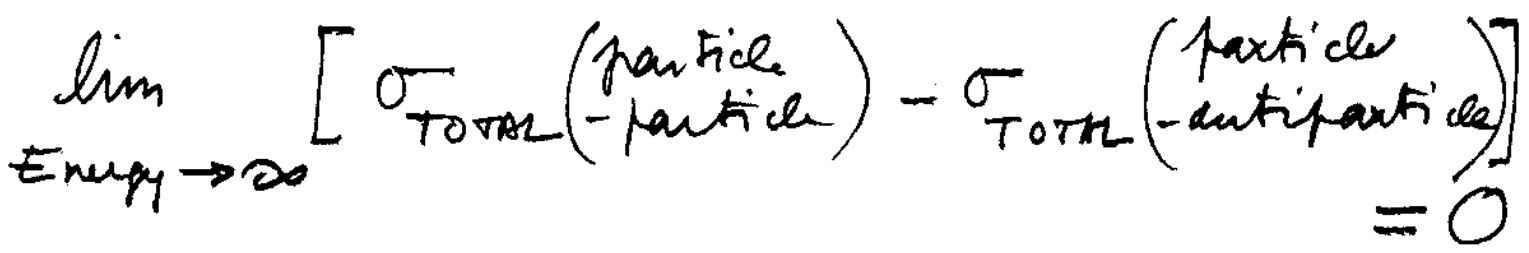

If the limit does not exist, zero belongs to the set of limiting values of the cross-section.

Our analogue is the following. Take the case of a potential gr of compact support, $\mathrm{v}$ being purely negative. Then, as noticed for instance by Ins and Simon, for $g \rightarrow-\infty$ the total cross-section tends to a limit which is the crosssection of a hard core. Incidentally, this limit is not necessarily reached monotonously, even though the phase shifts (Kate monotony) are monotonous functions of g. Anyway, the limit exists. The limit of the full amplitude exists as well. So we have

$$
F(g) \rightarrow C, \text { for } g \rightarrow-\infty \text {. }
$$

Intuitively, we have higher and higher obstacles as $\mathrm{g}$ increases, and in the end the detailed shape of the potential does not count, but only its support:
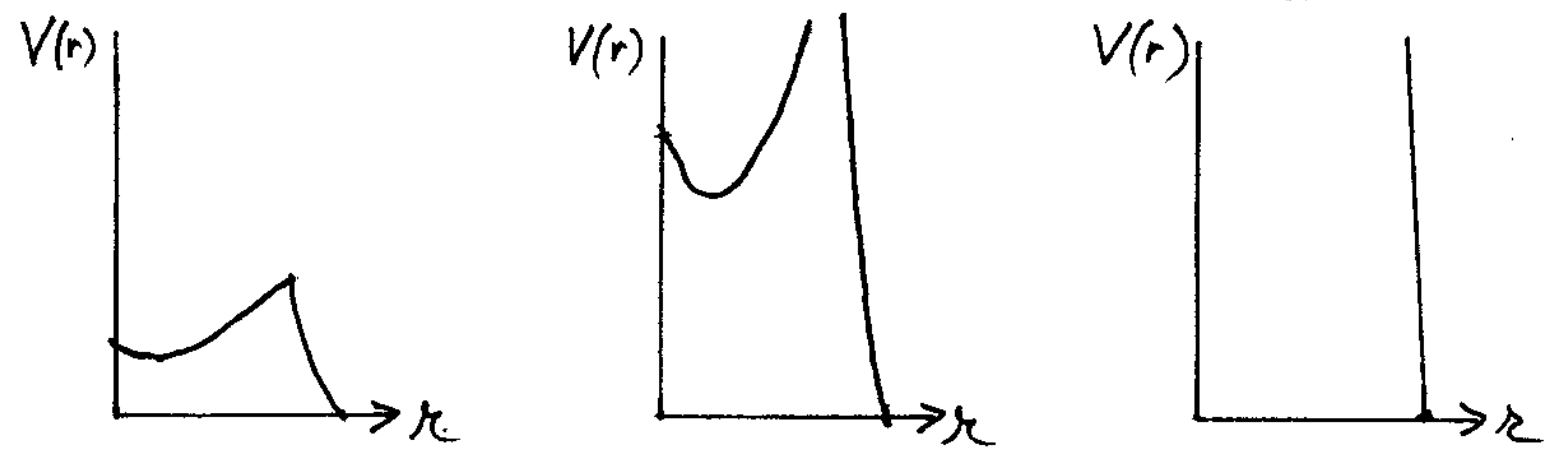

The question you can ask yourself is what happens for $g \rightarrow \infty$, i.e., when you have a deeper and deeper hole.

Let us first notice that if $F(g)$ is Herglotz and if $F(g) \rightarrow c$ for $g \rightarrow$ $\rightarrow-\infty \mathrm{F}(\mathrm{g}) \rightarrow c$ for $\mathrm{g} \rightarrow \infty$ in any complex direction. The proof is as follows:

$|\operatorname{exf} i F(g)|=\exp -I_{m} F(g) \leqslant 1$ 
So $\exp i F(g)$ is bounded in $\operatorname{Im} F(g)>0$ and has a limit for $g \rightarrow-\infty$. A small modification of Montel's theorem says that exp $\mathrm{iF}(\mathrm{g})$ has exactly the same limit in any complex direction. Naturally, if $F(g)$ has a limit for $g \rightarrow+\infty$, this Iimit is necessarily again the same and we would have

$$
\lim _{g \rightarrow+\infty}(\sigma(g)-\sigma(-g))=0
$$

In fact this is not so. $\operatorname{Lim} \sigma(g)$ for $g \rightarrow+\infty$ does not exist. However, we have a statement completely analogous to the Pomeranchuk theorem ${ }^{11)}$ :
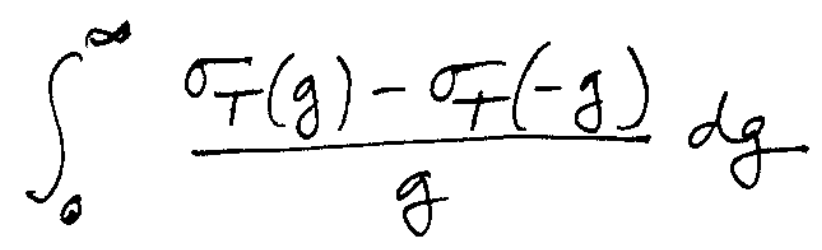

converges. Therefore, 0 belongs to the set of limiting values of $\sigma_{\mathrm{T}}(\mathrm{g})-$ - $\sigma_{T}(-g)$. Harald Grosse has checked that this is the case for a spherical square well. As $g \rightarrow+\infty$, one finds that $\sigma_{T}(g)$ stays very close to the hard core value except for some occasional spikes of the order of $g^{1 / 2}$.

It has been pointed out to me by Dr. Enss that, for a cubic well, one gets oscillations of the cross-section from zero to twice the hard core value as $g \rightarrow+\infty$. 


\section{REFERENCES}

1) W.O. Amrein and D.C. Pearson, J. Phys. A 12 (1979) 1469;

W.O. Amrein, D.C. Pearson and K.B. Sinha, Scattering theory in quantum mechanics, Reading, Benjamin (1977).

This paper contains references to previous work

2) V. Enss and B. Simon, Phys. Rev. Letters 44 (1980) 319 and 764 ;

V. Enss and B. Simon, to appear in Comm. Math. Phys.;

V. Enss and B. Simon, preprint to appear in Classical, Semi-Classical and Quantum Mechanical Problems in Mathematics, Chemistry and Physics, editors K. Gustafson and W.P. Reinhardt, Plenum (1980/81).

3) J.M. Combes and M. Guez, private communication, to be published.

4) M. Froissart, Phys. Rev. 123 (1961) 1053.

5) A. Martin, Nuovo Cimento 42 (1966) 930.

6) A. Martin, Lectures at the University of Washington, Seattle (1964), unpublished; See aiso A. Martin, Nuovo Cimento 23 (1962) 641;

A. Martin Nuovo Cimento, 31 (1964) 1229.

7) K. Chadan and A. Martin, appendix of Comm. Math. Phys. 70 (1979) 1.

8) A. Martin, Comm. Math. Phys. 69 (1979) 89; Notice that in the present lectures we give an improved version in which the total cross-section is bounded by $I^{3 / 2}$ for large $I$, instead of $I^{2}$.

9) A. Martin, Comm. Math. Phys. 73 (1980) 79. 10) J.A. Shohat and J.D. Tamarkin, "The problem of Moments", American Mathematical
Society, New York, (1943).

II) See for instance, A. Martin Nuovo Cimento 39 (1965) 704. 